Memoirs of the College of Science, University of Kyoto, Series A

Vol. XXXII, Mathematics No. 2, 1959.

\title{
Analyticité des solutions élémentaires du système hyperbolique à coefficients constants
}

Par

Sigeru Mrzohata

(Reçu le 31 août 1959)

1. Introduction. Soit $E(x, t)$ une solution élémentaire du système hyperbolique :

$$
\frac{\partial u}{\partial t}-\sum_{j=1}^{n} A_{j} \frac{\partial u}{\partial x_{j}}-B u=0,
$$

où $A_{j}$ et $B$ sont des matrices constantes d'ordre $N$.

Notre but est de montrer que $E(x, t), t \geqslant 0$, est une fonction analytique ${ }^{1)}$ sauf sur les lignes bicaractéristiques issues de l'origine. Ce résultat est déjà connu pour les systèmes homogènes, à savoir dans le cas où $B=0$ (Petrowsky [4]). Mais, ici, nous allons le montrer directement par le développement asymptotique de l'image de Fourier de $E(x, t)$. Nous suiverons le raisonnement de P. D. Lax exposé dans [1] pour montrer que $E(x, t)$ est une fonction indéfiniment différentiable sauf sur les bicaractéristiques issues de l'origine dans le cas où $A_{j}$ et $B$ sont des matrices indéfiniment différentiables. On pourrait étendre notre résultat au cas où $A_{j}$ et $B$ sont analytiques en $(x, t)$, mais il faudra alors une analyse très minutieuse.

2. Ce que nous appelons ici hyperbolique est le suivant (voir Petrowsky [3] p. 66) :

$1^{\circ}$ pour tout $\xi=\left(\xi_{1}, \cdots, \xi_{n}\right)$ réel,

$A \cdot \xi=\sum A_{j} \xi_{j}$ est une matrice diagonalisable dont les valeurs caractéristiques sont toutes réelles;

$2^{\circ}$. les multiplicités des valeurs caractéristiques sont invariantes pour $\xi$ :

1) Dans cet article, nous entendons par une fonction analytique une fonction analytique de variables réelles. 


$$
\begin{aligned}
& \operatorname{det}(\lambda I-A \cdot \xi)=\left(\lambda-\lambda_{1}(\xi)\right)^{p_{1}} \cdots\left(\lambda-\lambda_{s}(\xi)\right)^{p_{s}}, \\
& \lambda_{1}(\xi)<\cdots<\lambda_{s}(\xi), \quad p_{1}+\cdots+p_{s}=N .
\end{aligned}
$$

On sait alors que les $\lambda_{i}(\xi)$ sont des fonctions analytiques.

Montrons notre but pour le système strictement hyperbolique : On suppose les valeurs caractéristiques distinctes. Comme notre raisonnement est essentiellement le même que pour le système hyperbolique au sens généralisé, nous ajoutons, à la fin, des modifications à faire.

Nous allons envisager d'abord le cas où $B=0$ :

$$
\frac{\partial u}{\partial t}-\sum_{j=1}^{n} A_{j} \frac{\partial}{\partial x_{j}} u=0 \text {. }
$$

On désigne par $\omega \xi=\left(\omega_{1} \xi, \cdots, \omega_{n} \xi\right),|\omega|=1, \xi \geqslant 0$, les points de l'espace dual de $x$.

Soit $u(x, t)$ l'une des solutions élémentaires :

$$
u(x, 0)=\left|\begin{array}{c}
0 \\
\vdots \\
\delta_{x} \\
\vdots \\
0
\end{array}\right|
$$

La transformation de Fourier donne ${ }^{2)}$

$$
\left(\frac{\partial}{\partial t}-i \sum_{j} A_{j} \cdot \omega \xi\right) \hat{u}(\omega \xi, t)=0, \quad \hat{u}(\omega \xi, 0)=\left(\begin{array}{c}
0 \\
\vdots \\
1 \\
\vdots \\
0
\end{array}\right) .
$$

Soit $R_{i}(\omega)$ est le vecteur propre de longueur unité de la matrice $A \cdot \omega=\sum_{j} A_{j} \omega_{j}$ correspondant à la valeur propre $\lambda_{i}(\omega)$ :

$$
\left(\lambda_{i}(\omega) I-A \cdot \omega\right) R_{i}(\omega)=0 .
$$

Il est évident que les $R_{i}(\omega)(i=1,2, \cdots, N)$ sont des vecteurs définis sur la sphère tout entière, analytiques ${ }^{3)}$ sauf peut-être dans le cas

2) Ce que nous appelons ici l'image de Fourier est, dans la notation actuelle,

$$
\begin{aligned}
& \hat{u}(\omega \xi)=\int \exp (-i x \cdot \omega \xi) u(x) d x . \text { Alors, l'image réciproque devient } \\
& \left.u(x, t)=\frac{1}{2 \pi}\right)^{n} \int \exp (i x \cdot \omega \xi) \hat{u}(\omega \xi) \xi^{n-1} d \xi d \omega .
\end{aligned}
$$

3) La sphère $\Omega_{0}:|\omega|=1$, est muni d'une structure de varièté analytique : $\omega_{i}=\omega_{i}\left(s_{1}, \cdots, s_{n-1}\right)(i=1,2, \cdots, n) s$ étant les coordonnées d'une carte locale. $R_{i}(\omega)$ est alors un vecteur dont les composants sont des fonctions analytiques en $s$. Il en est de même de $\lambda_{i}(\omega), \sigma_{j}(\omega)$, etc. $\cdots$. 
$n=2$. $^{4} \quad$ Laissons ce cas de côté.

Comme les $R_{i}$ sont linéairement indépendants, on exprime la transformée de la donnée initiale:

$$
\left(\begin{array}{c}
0 \\
\vdots \\
1 \\
\vdots \\
0
\end{array}\right)=\sum_{j} \sigma_{j}(\omega) R_{j}(\omega) .
$$

Remarquons que $\sigma_{j}(\omega)$ sont également analytiques.

On a alors

$$
\hat{u}(\omega \xi, t)=\sum_{j=1}^{N} \exp \left(i \lambda_{j}(\omega) t \xi\right) \sigma_{j}(\omega) R_{j}(\omega)
$$

la solution voulue de $(2,1)$.

La solution $u(x, t)$ de $(1,1)^{\prime}$ s'écrit

$$
u(x, t)=(2 \pi)^{-n} \sum_{j} \int \exp \left[i\left(x \cdot \omega+t \lambda_{j}\right) \xi\right] \sigma_{j}(\omega) R_{j}(\omega) \xi^{n-1} d \xi d \omega
$$

où $d \omega$ désigne l'élément de volume de la sphère unité $\Omega_{0}$.

Il faut signaler que l'expression ci-dessus ne s'écrit que symboliquement pour exprimer l'image réciproque de Fourier, mais pour le calcul elle est commode.

Une courbe bicaractéristique $x(t)$ issue de l'origine de l'équation $(1,1)$ ou bien de $(1,1)^{\prime}$ est définie par

$$
x_{i}=-\lambda_{\omega_{i}}^{\prime}(\omega) t, t=t \quad(i=1,2, \cdots, n),
$$

où $\lambda_{\omega_{i}}^{\prime}(\omega)$ désigne la dérivation suivante : on étend $\lambda(\omega)$, pour $|\omega|>0$, par la relation $\lambda(\omega)=|\omega| \lambda\left(\frac{\omega_{1}}{|\omega|}, \cdots, \frac{\omega_{n}}{|\omega|}\right)$ et $\lambda_{\omega_{i}}^{\prime}=\left.\frac{\partial \lambda}{\partial \omega_{i}}\right|_{\left.\right|_{\omega \mid} \mid=1} \cdot \lambda(\omega)$ étant l'une des $\lambda_{j}(\omega)$. On désigne par $b_{j}(\omega)$ la ligne ci-dessus correspondant à $\lambda=\lambda_{j}$. Par $(B)$ l'ensemble des lignes bicaractéristiques issues de l'origine.

Soit $(x, t), t>0$, un point n'appartenant pas à $(B)$. On va montrer que $u(x, t)$ donnée par $(2,5)$ est une fonction analytique en $(x, t)$ (à valeurs vectorielles) au voisinage de ce point.

4) Dans le cas $n=2$, comme le cercle n'est pas homotopiquement 0 , il faut quelques considérations pour définir $R_{i}(\omega)$ sur tout cercle comme un vecteur continu. Mais, comme nous le montrerons dans le $n^{\circ} 4$, il nous suffit de supposer que $R_{i}(\omega)$ est analytique par morceau. 
Posons

$$
l(\omega ; x, t)=l(\omega)=x \cdot \omega+t \lambda_{j}(\omega) .
$$

Nous allons montrer que

$$
u(x, t)=\int_{\xi \geqq 1} \exp [i l(\omega ; x, t) \xi] \psi(\omega) \xi^{n-1} d \xi d \omega
$$

est une fonction analytique en $(x, t)$ au voisinage du point $(x, t)$ vérifiant la condition ci-dessus, où $\psi(\omega)$ est une fonction analytique en $\omega$. Cela suffit pour montrer notre but, car $\int_{|\xi| \leqslant 1}$ " $d \xi d \omega$ est une fonction analytique partout.

De $(2,7)$, pour $\nu=\left(\nu_{1}, \cdots, \nu_{n}, \nu_{n+1}\right)=\left(\nu^{\prime}, \nu_{n+1}\right)$, on a

$(2,8) \quad D^{\nu} u(x, t)=i^{|\nu|} \int_{\xi \geqslant 1} \exp (i l \xi) \omega^{\nu^{\prime}} \lambda_{j}^{\nu_{n+1}} \psi(\omega) \xi^{|\nu|+n-1} d \xi d \omega$.

Pour évaluer ces dérivées successives, nous suivons le raisonnement de P. D. Lax.

Le point de l'intersection $x_{0}$ de la ligne parallèle à $b_{j}(\omega)$ avec le plan $t=0$ est donné par

$$
x_{0}=x+\left(\lambda_{j}\right)^{\prime} t=\left(x_{1}+\left(\lambda_{j}\right)_{\omega_{1}}^{\prime} t, \cdots, x_{n}+\left(\lambda_{j}\right)_{\omega_{n}}^{\prime} t\right) .
$$

L'hypothèse que $(x, t)$ n'est pas situé sur aucune bicaractéristique issue de l'origine entraîne que

$$
\left|x_{0}\right|=\left|x+\lambda_{j}^{\prime} t\right|>0 \text {. }
$$

Or, $\frac{\partial l}{\partial \omega_{i}}=x_{i}+t\left(\lambda_{j}\right)_{\omega_{i}}^{\prime}$, d'où

$$
|\operatorname{grad} l(\omega)|=\left|x_{0}\right|>0 .
$$

Comme $\omega \cdot \operatorname{grad} l(\omega)=0$, en point $\omega$ tel que $l(\omega)=0$, on voit que la longueur du gradient de $l(\omega)$ suivant la surface $|\omega|=1$, est minoré par une constante positive lorsque $\omega$ parcourt la variété $V=\{\omega$; $l(\omega)=0\}$.

Considérons la sphère $|\omega|=1$ comme une variété riemannienne analytique, et $V$, définie par $l(\omega)=0$, comme une sous-variété analytique régulière à $(n-2)$ dimensions. Considérons le voisinage de $V$. Par chaque point de $V$, il passe une et une seule trajectoire orthogonale aux hypersurfaces $l(\omega)=$ constantes. Si l'on prend un voisinage de $V$ assez petit, alors pour chaque point, il en existe une 
et une seule. On munit $V$ des cartes locales analytiques $\left(u_{1}, \cdots\right.$, $\left.u_{n-2}\right)$. Comme pour tout point $P=\left(s_{1}, \cdots, s_{n-1}\right)$ il existe une seule trajectoire passant par $\left(u_{1}, \cdots, u_{n-2}\right)$, en désignant $l=l(P)$, on donne au point $P$, les coordonnées $\left(u_{1}, \cdots, u_{n-2}, l\right)$. Alors $\left(s_{1}, \cdots, s_{n-1}\right)$ sont des fonctions analytiques de $\left(u_{1}, \cdots, u_{n-2}, l\right)$ et la correspondance est biunivoque. En effet, la trajectoire orthogonale se donne comme solution de

$$
\frac{d s_{i}}{d \sigma}=\sum_{j} g^{i j}(s) l_{s_{j}}^{\prime}(s)
$$

où $\left(g^{i j}\right)$ est la matrice inverse de $\left(g_{i j}\right): d s^{2}=\sum g_{i j}(s) d s_{i} d s_{j}$. En prenant $l$ comme paramètre au lieu de $\sigma$,

$$
\frac{d s_{i}}{d l}=\frac{d s_{i}}{d \sigma} \frac{d \sigma}{d l}=\sum_{j} g{ }^{i j} l_{s_{j}}^{\prime} / \sum_{i, j} g{ }^{i j} l_{s_{i}}^{\prime} l_{s_{j}}^{\prime} \quad(i=1,2, \cdots, n-1) .
$$

Comme $g^{i j}(s)$ et $l(s)$ sont des fonctions analytiques, en désignant par $s_{i}(l ; u)$ la solution de $(2,11)$ vérifiant la condition initiale $s_{i}(0 ; u)=s_{i}(u)$, d'après la théorie des équations différentielles, on voit que $s_{i}(l ; u)$ sont des fonctions analytiques de $(l, u)=\left(l, u_{1}, \cdots, u_{n-2}\right)$, puisque $s_{i}(u)$ sont des fonctions analytiques de $u$.

On prend maintenant une partition de l'unité $\left\{\alpha_{i}(u)\right\}_{i=1,2, \cdots, p}$ sur $V$, indéfiniment différentiables $\sum \alpha_{i}(u) \equiv 1$ subordonnée aux cartes locales qu'on vient d'introduire, et $\beta(l)$ est indéfiniment différentiable à support petit et vaut 1 au voisinage de $l=0 ; 0 \leqslant \beta(l) \leqslant 1$. On fait l'orientation telle que $d u_{1} \cdots d u_{n-2} d l>0$.

Alors, $(2,8)$ s'écrit

$$
\begin{aligned}
D^{\nu} u(x, t)= & \int_{\xi \geqslant 1} \xi^{|\nu|+n-1} d \xi \int \exp (i l \xi) \sum_{i} \beta(l) \alpha_{i}(u) \psi_{\nu}(\omega) \\
& \times \frac{\partial\left(s_{1}, \cdots, s_{n-1}\right)}{\partial\left(u_{1}, \cdots, u_{n-2}, l\right)} \mathcal{P}_{i}(s) d u_{1} \cdots d u_{n-2} d l \\
& +\int_{\xi \geqslant 1} \xi^{|\nu|+n-1} d \xi \int \exp (i l \xi)[1-\beta(l)] \psi_{\nu}(\omega) d \omega,
\end{aligned}
$$

où $\psi_{\nu}(\omega)=\omega^{\nu^{\prime}} \lambda_{j}^{\nu_{n+1}} \psi(\omega), \Phi_{i}(s)$ est une fonction analytique en $s$.

Nous avons supposé $(x, t)$ fixé. Maintenant supposons que $(x, t)$ parcourt un petit voisinage $U$ de $\left(x_{0}, t_{0}\right)$. Montrons comment $(x, t)$ intervient dans la paramétrisation précédente. D'abord, $l(s ; x, t)=0$, $l_{s}^{\prime}\left(s ; x_{0}, t_{0}\right) \neq 0$ entraîne que, si $U$ est assez petit, on a, par exemple $s_{n-1}=f\left(s_{1}, \cdots, s_{n-2} ; x, t\right)$ où $f$ est une fonction analytique en $(s, x, t)$. Ceci signifie que, si $U$ est assez petit, la sous-variété $V_{x, t}=\{s$; 
$l(s ; x, t)=0\}$ s'exprime par $s_{i}=s_{i}\left(u_{1}, \cdots, u_{n-2} ; x, t\right) \quad(i=1,2, \cdots, n-2)$ où $s_{i}$ sont des fonctions analytiques en $(u, x, t)$. D'après la formule $(2,11)$, au voisinage de $V_{x, t}, s_{i}(i=1, \cdots, n-1)$ sont représentés biunivoquement par $\left(u_{1}, \cdots, u_{n-2}, l\right)$. Précisément, $s_{i}=s_{i}\left(u_{1}, \cdots\right.$, $\left.u_{n-2} ; x, t\right)$. Pour $(x, t)$ fixé dans $U,\left(u_{1}, \cdots, u_{n-2}, l\right) \rightarrow\left(s_{1}, \cdots, s_{n-1}\right)$ est biunivoque. De plus $s_{i}$ sont des fonctions analytiques en $(u, l ; x, t)$. Alors toute fonction analytique en $s$ se transforme en une fonction analytique en $\left(u_{1}, \cdots, u_{n-2}, l, x, t\right)$.

Rappelons que la distribution $(2,7)$ s'exprime à l'aide du facteur de convergence $\exp (-\varepsilon \xi), \varepsilon>0$.

$$
u(x, t)=\lim _{\varepsilon \rightarrow 0} \int \exp (i l \xi-\varepsilon \xi) \psi(\omega) \xi^{n-1} d \xi d \omega .
$$

Cette limite est à prendre au sens de distribution $\left(\mathfrak{D}^{\prime}\right)_{x}$. D'où, il en est de même de la distribution $(2,12)$. Nous allons montrer que $D^{\nu} u(x, t)$ est une fonction continue sur le voisinage $U$ de $\left(x_{0}, t_{0}\right)$ et cette vérification montre en même temps son évaluation sur $U$. Envisageons d'abord le second terme.

$$
\begin{gathered}
\int_{\xi \geqslant 1} \xi^{m+n-1} \exp (i l \xi-\varepsilon \xi) d \xi=\exp (i l-\varepsilon)\left\{-\frac{1}{i l-\varepsilon}+\frac{m+n-1}{(i l-\varepsilon)^{2}}\right. \\
-\frac{(m+n-1)(m+n-2)}{(i l-\varepsilon)^{3}}+\cdots+(-1)^{m+n-1} \frac{(m+n-1) !}{(i l-\varepsilon)^{m+n-1}} \\
\left.+(-1)^{m+n} \frac{(m+n-1) !}{(i l-\varepsilon)^{m+n}}\right\},
\end{gathered}
$$

Compte tenu de ce que $|l| \geqslant \delta^{\prime}$, où $\beta(l) \neq 1$, on a

$$
\begin{aligned}
& \lim _{\varepsilon \rightarrow 0} \int_{\Omega_{0}} \frac{\exp (i l-\varepsilon)}{(i l-\varepsilon)^{k}}[1-\beta(l)] \psi_{\nu}(\omega) d \omega \\
& \quad=\frac{1}{i^{k}} \int_{\Omega_{0}} \frac{\exp [i l(\omega ; x, t)]}{l(\omega ; x, t)^{k}}[1-\beta(l)] \psi_{\nu}(\omega) d \omega,
\end{aligned}
$$

la convergence est uniforme pour $(x, t) \in U$. D'où

$(2,15) \quad$ second terme de $(2,12) \mid$

$$
\begin{aligned}
& \leqslant\left[\frac{1}{\delta^{\prime}}+\frac{m+n-1}{\delta^{\prime 2}}+\cdots+\frac{(m+n-1) !}{\delta^{\prime m+n}}\right] \int_{\Omega_{0}}\left|\psi_{\nu}(\omega)\right| d \omega \\
& \leqslant \frac{(m+n) !}{\delta^{\prime m+n}} \int_{\Omega_{0}}\left|\psi_{\nu}(\omega)\right| d \omega, \quad \text { où } m=|\nu| .
\end{aligned}
$$


Envisageons le premier terme; on considère

$$
\begin{aligned}
& \psi_{i}^{(\nu)}(x, t)=\int_{\xi \geqslant 1} \xi^{|\nu|+n-1} d \xi \int \exp (i l \xi) \beta(l) \alpha_{i}(u) \psi_{\nu}(\omega) \\
& \quad \times \frac{\partial\left(s_{1}, \cdots, s_{n-1}\right)}{\partial\left(u_{1}, \cdots, l\right)} \rho_{i}(s) d u_{1} \cdots d u_{n-2} d l
\end{aligned}
$$

Il faut, bien entendu, calculer à l'aide du facteur de convergence $\exp (-\varepsilon \xi)$. Mais nous l'omettons ici, car la vérification est facile d'après ce qui précède.

Le facteur $\frac{\partial\left(s_{1} \cdots\right)}{\partial\left(u_{1} \cdots\right)} \varphi_{i}(s)$ est une fonction analytique en $\left(u_{1}, \cdots\right.$, $\left.u_{n-2}, l ; x, t\right)$. Alors il existe des constantes positives $M_{1}, \cdots, M_{n+1}$, $M_{\psi}, A$ et $\rho$ telles que

$$
\begin{gathered}
\left|\left(\frac{\partial}{\partial l}\right)^{k}\left[\psi_{\nu}(\omega) \frac{\partial\left(s_{1} \cdots\right)}{\partial\left(u_{1} \cdots\right)} \rho_{i}(s)\right]\right| \leqslant{ }_{\rho^{k}}^{k !} M_{1}^{\nu_{1}} \cdots M_{n+1}^{\nu_{n+1}} M_{\psi} A, \\
k=0,1,2, \cdots ; \nu \geqslant 0, \quad \text { pour } \quad(x, t) \in U .
\end{gathered}
$$

(Ici, il faut remarquer que $s_{i}$ sont des fonctions analytiques en $(u, l ; x, t))$.

Ou bien, en désignant par $M=\max \left(M_{1}, \cdots, M_{n+1}\right), q_{\nu}(u, l ; x, t)$ $=\psi_{\nu}(\omega) \frac{\partial\left(s_{1} \cdots\right)}{\partial\left(u_{1} \cdots\right)} \phi_{i}(s)$,

$(2,16)^{\prime} \quad\left|\left(\frac{\partial}{\partial l}\right)^{k} q_{\nu}(u, l ; x, t)\right| \leqslant \frac{k !}{\rho^{k}} M^{|\nu|} M_{\psi} A, \quad$ pour $\quad(x, t) \in U$.

L'intégation par parties en $l$ donne

$$
\begin{aligned}
& \int \exp (i l \xi) \beta(l) \alpha_{i}(u) q_{\nu}(u, l) d u d l \\
& \quad=\frac{(-i)^{m+n+1}}{\xi^{m+n+1}} \int \exp (i l \xi) \alpha_{i}(u)\left(\frac{\partial}{\partial l}\right)^{m+n+1}\left[\beta(l) q_{\nu}(u, l)\right] d u d l .
\end{aligned}
$$

Or, $\quad\left(\frac{\partial}{\partial l}\right)^{m+n+1}\left[\beta(l) q_{\nu}(u, l)\right]=\beta(l)\left(\frac{\partial}{\partial l}\right)^{m+n+1} q_{\nu}(u, l)$

$$
+\sum_{p \neq 0} C_{p}^{m+n+1}\left(\frac{d}{d l}\right)^{p} \beta(l)\left(\frac{\partial}{\partial l}\right)^{m+n+1-p} q_{\nu}(u, l) .
$$

Envisageons les termes sous le signe $\Sigma$ : 


$$
\begin{aligned}
& \int \exp (i l \xi) \alpha_{i}(u)\left(\begin{array}{c}
\partial \\
\partial l
\end{array}\right)^{p} \beta \cdot\left(\frac{\partial}{\partial l}\right)^{m+n+1-p} q_{\nu}(u, l) d u d l \\
& =(-1)^{p} \int \alpha_{i}(u) \beta(l)\left(\frac{\partial}{\partial l}\right)^{p}\left[\exp (i l \xi)\left(\frac{\partial}{\partial l}\right)^{m+n+1-p} q_{\nu}(u, l)\right] d u d l \\
& =(-1)^{p} \sum_{k \leqslant p} C_{k}^{p}(i \xi)^{k} \int \alpha_{i}(u) \beta(l) \exp (i l \xi)\left(\frac{\partial}{\partial l}\right)^{m+n+1-k} q_{\nu}(u, l) d u d l
\end{aligned}
$$

Ces termes interviennent dans l'intégaration envisagée sous la forme (à puissances de $i$ près)

$$
\int_{\xi>1} \xi^{k-2} d \xi \int \exp (i l \xi) \alpha_{i}(u) \beta(l)\left(\frac{\partial}{\partial l}\right)^{m+n+1-k} q_{\nu}(u, l) d u d l .
$$

Ici, il faut souligner que l'intégration en $d u d l$ s'étend sur le domaine du $(u, l)$ vérifiant $\beta^{\prime}(l) \neq 0$. Donc, dans ce domain $|l| \geqslant \delta^{\prime}$.

Or, d'après ce qui précède, on voit facilement que cette intégrale se majore par

$$
\frac{2 \cdot k !}{\delta^{\prime k}} \int\left|\left(\begin{array}{c}
\partial \\
\partial l
\end{array}\right)^{m+n+1-k} q_{\nu}(u, l)\right| d u d l \leqslant \frac{2 \cdot k !}{\delta^{\prime k}} \frac{(m+n+1-k) !}{\rho^{m+n+1-k}} M^{|\nu|} M_{\psi} A S_{i},
$$

de là

$$
\begin{aligned}
& \left|\psi_{i}^{(\nu)}(x, t)\right| \leqslant M^{|\nu|} M_{\psi} A S_{i} \\
& \quad \times\left\{\frac{(m+n+1) !}{\rho^{m+n+1}}+\sum_{p \neq 0} C_{p}^{m+n+1}\left(\sum_{k<p} C_{k}^{n} 2 \frac{k !}{\delta^{\prime k}} \frac{(m+n+1-k) !}{\rho^{m+n+1-k}}\right)\right\} .
\end{aligned}
$$

On désigne $\rho^{\prime}=\min \left(\delta^{\prime}, \rho\right)$. Alors,

$$
\begin{aligned}
\sum_{k \leqslant p} \cdots & \leqslant \frac{2 \cdot p !}{\rho^{\prime m+n+1}} \sum_{k \leqslant p} \frac{(m+n+1-k) !}{(p-k) !} \leqslant \frac{2 \cdot p !}{\rho^{\prime m+n+1}} \cdot \frac{(m+n+1) !}{p !}(p+1)^{5)} \\
& \leqslant 2 \frac{(p+1) \cdot(m+n+1) !}{\rho^{\prime m+n+1}} \leqslant 2 \frac{(m+n+2) !}{\rho^{\prime m+n+1}} .
\end{aligned}
$$

D’où, finalement,

$$
\left|\psi_{i}^{(\nu)}(x, t)\right| \leqslant \frac{M^{|\nu|} M_{\psi} A S_{i}}{\rho^{\prime m+n+1}}(m+n+2) ! 2^{m+n+2}, \quad m=|\nu| .
$$

En ajoutant cette inégalité par $i$, et compte tenu de $(2,15)$, on aura l'inégalité de la forme

5) $\frac{(m+n+1-k) !}{(p-k) !} \leqslant \frac{(m+n+1) !}{p !}$ 
$(2,18) \quad\left|D^{\nu} u(x, t)\right| \leqslant \frac{2^{|\nu|+n+2}}{\rho^{\prime|\nu|+n+1}} M^{\prime \nu \mid} M_{\psi}^{\prime} k\left|\Omega_{0}\right| \cdot(|\nu|+n+2) ! \quad$ pour $(x, t) \in U$, où $M^{\prime}$ est une constante ne dépendant que de $\omega$ et $\lambda_{i}(\omega) \quad(i=1,2$, $\cdots, N)$, et $M_{\psi}^{\prime}$ ne dépend que de $\psi(\omega), k$ est une constante $\geqslant 2$ et $\left|\Omega_{0}\right|$ est la mesure superficielle de $\Omega_{0}$.

L'évaluation $(2,18)$ montre que la distribution définie par $(2,7)$ est une fonction analytique en $(x, t)$ au voisinage du point $\left(x_{0}, t_{0}\right)$ n'appartenant pas à $(B)$. Cela achève notre démonstration.

3. Dans ce numéro, nous allons envisager le cas général, à savoir le cas où $B=1=0$, en supposant les racines $\lambda_{j}(\omega)$ réelles et distinctes.

Posons

$$
\hat{u}(\omega \xi, t)=\sum_{j=1}^{N} \exp \left(i \lambda_{j}(\omega) \xi t\right)\left\{v_{0}^{(j)}(\omega, t)+\frac{v_{1}^{(j)}(\omega, t)}{\xi}+\cdots+\frac{v_{n}^{(j)}(\omega, t)}{\xi^{n}}+\cdots\right\} .
$$

Notre premier but est de montrer que, si $\xi$ est assez grand, cette série uniformément (par rapport à $\omega, t$ ) converge.

Suivons le procédé montré par P. D. Lax dans [1].

$$
\begin{aligned}
& \left(\frac{\partial}{\partial t}-\sum_{j} A_{j} \frac{\partial}{\partial x_{j}}-B\right) u(x, t)=0 \quad \text { équivaut à } \\
& \left(\frac{\partial}{\partial t}-i A \cdot \omega \xi-B\right) \hat{u}(\omega \xi, t)=0 .
\end{aligned}
$$

Ceci entraîne

$$
\begin{aligned}
& \left(\lambda_{i}(\omega)-A \cdot \omega\right) v_{0}^{(i)}=0 \quad(i=1,2, \cdots, N) ; \\
& \left(\lambda_{i}(\omega)-A \cdot \omega\right) v_{n}^{(i)}=-\sqrt{-1}\left\{\left(v_{n-1}^{(i)}\right)_{t}^{\prime}-B v_{n-1}^{(i)}\right\} \\
& n=1,2, \cdots ; i=1,2, \cdots, N .
\end{aligned}
$$

Posons

$$
B R_{i}(\omega)=\sum_{j} b_{i j}(\omega) R_{j}(\omega)
$$

Remarquons que les $b_{i j}(\omega)$ sont des fonctions analytiques.

Posons

$$
v_{n}^{(i)}=\sum_{j} \sigma_{n}^{i j}(\omega, t) R_{j}(\omega)
$$

Alors

$$
B v_{n}^{(i)}=\sum_{j} \sigma_{n}^{i j} B R_{j}=\sum_{j, k} \sigma_{n}^{i j} b_{j k} R_{k}=\sum_{j}\left(\sum_{k} \sigma_{n}^{i k} b_{k \jmath}\right) R_{j} .
$$

L'équation $(3,2)$ devient 


$$
\sigma_{n}^{i j}(\omega, t)=\frac{\sqrt{-1}}{\lambda_{j}-\lambda_{i}}\left\{\left(\sigma_{n-1}^{i j}\right)_{t}^{\prime}-\sum_{k} \sigma_{n-1}^{i k} b_{k j}\right\} \quad i \neq j ;
$$

$$
\left(\sigma_{n-1}^{i i}\right)_{t}^{\prime}-\sum_{k} \sigma_{n-1}^{i k} b_{k i}=0 \quad \text { ou bien }
$$

$$
\left(\sigma_{n-1}^{i i}\right)_{t}^{\prime}-b_{i i} \sigma_{n-1}^{i i}=\sum_{k \neq i} \sigma_{n-1}^{i k} b_{k i} .
$$

D'après la condition $(3,1)$, on pose

$$
v_{0}^{(i)}=\sigma_{0}^{(i)}(\omega, t) R_{i}(\omega),
$$

alors la condition $(3,6)^{\prime}$ implique

$(3,6)_{0} \quad\left(\sigma_{0}^{(i)}\right)_{t}^{\prime}-b_{i i} \sigma_{0}^{(i)}=0 \quad$ avec la condition initiale

$$
\sigma_{0}^{(i)}(\omega, 0)=\sigma_{i}(\omega) \quad(i=1,2, \cdots, N) .
$$

Ensuite, pour $v_{n}^{(i)}(\omega, t)$ on pose la codition initiale

$$
v_{n}^{(i)}(\omega, 0)=0 \quad n=1,2, \cdots .
$$

Cette condition équivaut à

$$
\sum_{i} \sigma_{n}^{i j}(\omega, 0)=0 \quad(j=1,2, \cdots, N) \quad(n=1,2, \cdots),
$$

ou bien

$$
\sigma_{n}^{i i}(\omega, 0)=-\sum_{j \mp i} \sigma_{n}^{j i}(\omega, 0) .
$$

Pour évaluer $\left(\frac{\partial}{\partial t}\right)^{p} \sigma_{n}^{i j}(\omega, t)$, commençons par

LEMME 3,1. Soit $x(t)$ la solution de l'équation différentielle $x^{\prime}-d x$ $=k(t)$ ou $d$ est une constante. On a pour $t \geqslant 0$,

$$
\begin{aligned}
& |x(t)| \leqslant a\left(|x(0)|+\max _{0 \leqslant i \leqslant t}|k(\tau)|\right) \quad \text { où } \quad a=\exp (|d| t) ; \\
& x^{(p)}(t)=d^{p} \cdot x(t)+\sum_{q=0}^{p-1} d^{p-q-1} k^{(q)}(t) \quad p \geqslant 1 .
\end{aligned}
$$

Ceci remarqué, désignons

$$
\begin{aligned}
& M_{n}^{(p)}=\max _{i, i=1, \cdots, N}\left\{\sup _{\omega \in \Omega_{0}, 0 \leqslant t \leqslant T}\left|\left(\frac{\partial}{\partial t}\right)^{p} \sigma_{n}^{i j}(\omega, t)\right|\right\}, \\
& M_{n}^{\prime(p)}=\max _{i \neq j}\left\{\sup \left|\left(\frac{\partial}{\partial t}\right)^{p} \sigma_{n}^{i j}(\omega, t)\right|\right\}, \\
& M_{n}^{(p)}=\max _{i=1,2, \cdots, N}\left\{\sup \left|\left(\frac{\partial}{\partial t}\right)^{p} \sigma_{n}^{i i}(\omega, t)\right|\right\}
\end{aligned}
$$




$$
\begin{aligned}
& \delta \leqslant \min _{\omega \in \Omega_{0}}\left|\lambda_{i}(\omega)-\lambda_{j}(\omega)\right| \quad i \neq j, \text { de plus } \delta<1, \\
& K=\max _{i, j}\left\{\max _{\omega \in \Omega_{0}}\left|b_{i j}(\omega)\right|\right\} ; \quad a=\exp (K T) .
\end{aligned}
$$

On a alors, d'après $(3,5)$

$$
\dot{M}_{n}^{\prime(p)} \leqslant \frac{1}{\delta}\left(M_{n-1}^{\prime(p+1)}+N K M_{n-1}^{(p)}\right) .
$$

D'après $(3,6)^{\prime},(3,7)$ et le lemme 3,1 , on a

$$
\begin{aligned}
& M_{n}^{(0)} \leqslant a\left(N M_{n}^{(0)}+N K M_{n}^{(0)}\right)=a(1+K) N M_{n}^{(0)}, \\
& { }^{\prime} M_{n}^{(p)} \leqslant K^{p} M_{n}^{(0)}+\sum_{q=0}^{p-1} K^{p-q-1} N K M_{n}^{\prime(q)} .
\end{aligned}
$$

On a alors le

LEMME 3, 2.

$$
M_{n}^{(p)} \leqslant c_{1}^{n}(c N K)^{n+p}\left(\frac{a}{\delta}\right)^{n+1} M,
$$

où $M=\max _{i=1,2, \cdots, N^{*}}\left\{\max _{\omega \in \Omega_{0}}\left|\sigma_{i}(\omega)\right|\right\}$; les $\sigma_{i}(\omega)$ étant définies par $(2,4) ; c$ est une constante positive quelconque $\geqslant 2 ; c_{1}$ est aussi une constante quelconque vérifiant $c_{1} \geqslant 2(1+K) N \frac{c+1}{c}$.

DÉmonstration. D'après $(3,6)_{0}$, l'inégalité à démontrer est vraie pour $n=0$. Nous allons montrer par récurrence en $n$. Supposons l'inégalité vraie pour $n-1$, montrons-la pour $n$.

D'après $(3,8)$,

$$
\begin{aligned}
M_{n}^{(p)} & \leqslant \frac{1}{\delta}\left(M_{n-1}^{(p+1)}+N K M_{n-1}^{(p)}\right) \\
& \leqslant \frac{1}{\delta} c_{1}^{n-1}(c N K)^{n+p-1}(c+1) N K\left(\frac{a}{\delta}\right)^{n} M .
\end{aligned}
$$

D'après la condition sur $c_{1}$, on a $(c+1) \leqslant c c_{1}$, et $a \geqslant 1$, de là l'inégalité voulue. Passons à ' $M_{n}^{(p)}$. D'abord d'après $(3,9)$ et $(3,11)$, on a

$$
{ }^{\prime} M_{n}^{(0)} \leqslant a(1+K) N c_{1}^{n-1}(c N K)^{n-1}(c+1) N K \frac{1}{\delta}\left(\frac{a}{\delta}\right)^{n} M,
$$

d'aprés la condition sur $c_{1},(1+K) N(c+1) \leqslant c c_{1}$, de là

$$
{ }^{\prime} M_{n}^{(0)} \leqslant c_{1}^{n}(c N K)^{n}\left(\frac{a}{\delta}\right)^{n+1} M .
$$


Ensuite, d'après $(3,10)$ et $(3,11)$

$$
\begin{aligned}
& { }^{\prime} M_{n}^{(p)} \leqslant K^{p \prime} M_{n}^{(0)}+N K \sum_{q=0}^{p-1} K^{p-q-1} c_{1}^{n-1}(c N K)^{n+q-1}(c+1) N K\left(\frac{a}{\delta}\right)^{n+1} M, \\
& \quad p \geqslant 1 . \\
& \text { D'abord, }
\end{aligned}
$$

$$
K^{p} M_{n}^{(0)} \leqslant c_{1}^{n} K^{p}(c N K)^{n}\left(\frac{a}{\delta}\right)^{n+1} M, \quad \text { comme } \quad c \geqslant 2,
$$

a fortiori $c N \geqslant 2$, de là $K^{p \prime} M_{n}^{(0)} \leqslant \frac{1}{2} c_{1}^{n}(c N K)^{n+p}\left(\frac{a}{\delta}\right)^{n+1} M$.

Ensuite, le second terme sous le signe $\Sigma$ devient

$$
c_{1}^{n-1}(c N K)^{n-1}(c+1)(N K)^{2}\left(\frac{a}{\delta}\right)^{n+1} M \sum_{q=0}^{p-1} K^{p-q-1}(c N K)^{q} ;
$$

compte tenu de ce que $\sum_{q=0}^{p-1} K^{p-q-1}(c N K)^{q}=K^{p-1}\left\{1+c N+\cdots+(c N)^{p-1}\right\}$ $\leqslant 2(c N K)^{p-1}$, puisque $c N \geqslant 2$, de là il se majore par $2 c_{1}^{n-1}(c N K)^{n+p-2}(c+1)(N K)^{2}\left(\frac{a}{\delta}\right)^{n+1} M$.

D'après la condition sur $c_{1}$ et $c$, on a $2(c+1) \leqslant \frac{1}{2} c_{1} c^{2}$, d'où le dernier se majore par $\frac{1}{2} c_{1}^{n}(c N K)^{n+p}\left(\frac{a}{\delta}\right)^{n+1} M$. Cela achève notre démonstration.

Dans le lemme 3,2, nous avons évalué en supposant $\omega$ réels. Mais, comme on s'aperçoit aisément, notre évaluation est même vraie dans un domaine complexe contenant les points réels. A savoir, on a le

LEMME 3, 3. Soit $(s)=\left(s_{1}, \cdots, s_{n-1}\right)$ une carte locale analytique de $\Omega_{0}$. Pour toute partie compacte $K_{s}$ des $(s)$, il existe une constante $\tilde{\rho}>0$ telle que $\sigma_{m}^{i j}(\omega(s), t), 0 \leqslant t \leqslant T(m=0,1,2, \cdots)$ peuvent $\hat{e} t r e$ prolongées analytiquement dans les polydisques de rayon $\tilde{\rho}$ ayant comme centre les points (réels) de $K_{s}$. De plus on y a l'inégalité de la forme du lemme 3,2:

$\left|\left(\frac{\partial}{\partial t}\right)^{p} \sigma_{m}^{i j}\left(\omega\left(s+i s^{\prime}\right), t\right)\right| \leqslant C^{m+p} M^{\prime}, \quad$ pour $\left|s_{i}^{\prime}\right| \leqslant \tilde{\rho} \quad(i=1,2, \cdots, n)$, $s \in K_{s}, \quad m=0,1,2, \cdots \quad p=0,1,2, \cdots$.

On va envisager maintenant

$(3,12) \quad u(x, t)=\sum_{j=1}^{N} \int_{\xi \geqslant \xi_{0}} \xi^{n-1} d \xi \int_{\Omega_{0}} \exp \left(i l_{j} \xi\right)\left\{v_{0}^{(j)}(\omega, t)+\cdots\right.$

$$
\left.+v_{m}^{(j)}(\omega, t) / \xi^{m}+\cdots\right\} d \omega \text {, }
$$


où $l_{j}=x \cdot \omega+t \lambda_{j}(\omega)$, et $\xi_{0}$ est un nombre assez grand qu'on va déterminer. Compte tenu de $(3,4)$, il suffit d'envisager une distribution de la forme:

$$
\begin{aligned}
u(x, t)= & \int_{\xi \geqslant \xi_{0}} \xi^{n-1} d \xi \int_{\Omega_{0}} \exp \left(i l_{j} \xi\right)\left\{\sigma_{0}^{(j)}(\omega, t)+\sigma_{1}^{j k}(\omega, t) / \xi+\cdots\right. \\
& \left.+\sigma_{m}^{j k}(\omega, t) / \xi^{m}+\cdots\right\} \psi(\omega) d \omega
\end{aligned}
$$

où $\psi(\omega)$ est une fonction analytique en $\omega$.

Posons

$$
\begin{aligned}
& u_{m}(x, t)=\int_{\xi \geqslant \xi_{0}} \xi^{n-1} d \xi \int \exp (i l \xi) \frac{\psi_{m}(\omega, t)}{\xi^{m}} d \omega \\
& \text { où } l=l_{j}, \psi_{m}=\sigma_{m}^{j k} \psi .
\end{aligned}
$$

$$
u(x, t)=u_{0}(x, t)+u_{1}(x, t)+\cdots+u_{m}(x, t)+\cdots \cdots .
$$

Prenons $\left(x_{0}, t_{0}\right)$ n'appartenant pas à $(B)$. Comme au $n^{0} 3$, on considère $u_{m}(x, t)$ au voisinage $U$ de $\left(x_{0}, t_{0}\right)$. La dérivation de $u_{m}(x, t)$ donne $^{6)}$ pour

$$
\begin{gathered}
D^{\nu}=\left(\frac{\partial}{\partial x_{1}}\right)^{\nu_{1}} \cdots\left(\frac{\partial}{\partial x_{n}}\right)^{\nu_{n}}\left(\frac{\partial}{\partial t}\right)^{\nu_{n+1}}, \nu=\left(\nu_{1}, \cdots, \nu_{n}, \nu_{n+1}\right)=\left(\nu^{\prime}, \nu_{n+1}\right) \\
(3,16) \quad D^{\nu} u_{m}(x, t)=\sum_{r} C_{r}^{\nu_{n+1}} \int_{\xi \geqslant \xi_{0}} \xi^{n-1+\left|\nu^{\prime}\right|+r} d \xi \\
\times \int \frac{\exp (i l \xi)(i \omega)^{\nu^{\prime}}\left(i \lambda_{j}\right)^{r}\left(\frac{\partial}{\partial t}\right)^{\nu_{n+1^{-r}}} \psi_{m}(\omega, t)}{\xi^{m}} d \omega .
\end{gathered}
$$

Nous utiliserons ici la paramétrisation $\left(u_{1}, \cdots, u_{n-2}, l\right)$ comme au $\mathrm{n}^{\circ} 2$. D'après le lemme 3,3 , correspondant à $(2,16)$, on aura l'inégalité de la forme:

$$
\begin{aligned}
& \left|\left(\frac{\partial}{\partial l}\right)^{k}\left\{\omega_{1}^{\nu_{1}} \cdots \omega_{n}^{\nu_{n}} \lambda_{j}^{\nu_{n+1}}\left(\frac{\partial}{\partial t}\right)^{p} \psi_{m}(\omega, t) \frac{\partial\left(s_{1} \cdots\right)}{\partial\left(u_{1} \cdots\right)} \rho_{i}(s)\right\}\right| \\
& \leqslant \frac{k !}{\rho^{k}} M^{|\nu|} M_{\sigma}^{m+p} A . \quad \nu \geqslant 0, \quad p, m=0,1,2, \cdots .
\end{aligned}
$$

Considérons d'abord le cas où $|\nu|+n \geqslant m$, alors dans $(3,16)$ $\left|\nu^{\prime}\right|+r \leqslant|\nu|$, d'où $n-1+\left|\nu^{\prime}\right|+r-m \geqslant|\nu|+n-1-m$. Donc, correspondant à $(2,18)$, on aura l'inégalité de la forme:

6) Si l'on utilise le théorème de Cauchy-Kowalewsky combiné avec le théorème de Holmgren sur l'unicité, il nous suffira de montrer seulement l'analyticité en $x$. Dans ce cas, le raisonnement sera plus bref. 
$(3,18)$

$$
\left|D^{\nu} u_{m}(x, t)\right| \leqslant 2^{|\nu|} \frac{2^{|\nu|+n+2-m}}{\left(\frac{\rho^{\prime}}{\xi_{0}}\right)^{|\nu|+n+1-m}} M^{\prime \nu \mid+m}(|\nu|+n+2-m) ! k\left|\Omega_{0}\right|
$$

pour $m \leqslant|\nu|+n, \quad(m=0,1,2, \cdots ; \nu \geqslant 0)$, où $M^{\prime}$ est supposé $\geqslant 1$. simple,

D'autre part, pour $|\nu|+n \leqslant m-1$, on a par l'évaluation très

$$
\left|D^{\nu} u_{m}(x, t)\right| \leqslant 2^{|\nu|} \frac{M^{\prime \nu \mid+m}}{\xi_{0}^{m-n-|\nu|}}
$$

On prend

$$
\xi_{0} \geqslant 2 M^{\prime}
$$

Maintenant envisageons

$$
\left|D^{v} u(x, t)\right| \leqslant \sum_{m=0}^{\infty}\left|D^{v} u_{m}(x, t)\right|=\sum_{m=0}^{|v|+n} \cdots+\sum_{m=|v|+n+1}^{\infty} \cdots .
$$

Dans la première somme, on majore tous les termes par (d'après $(3,18))$

$$
2^{|\nu|} \frac{2^{|\nu|+n+2}}{\left(\frac{\rho^{\prime}}{\xi_{0}}\right)^{|\nu|+n+1}} M^{\prime 2|\nu|+n}(|\nu|+n+2) ! k\left|\Omega_{0}\right| .
$$

D'autre part, le second membre se majore d'après $(3,20)$ par

$$
2^{|\nu|} 2 \frac{M^{\prime 2|\nu|+n+1}}{\xi_{0}}\left|\Omega_{0}\right|
$$

D'où

$(3,21) \quad\left|D^{\nu} u(x, t)\right| \leqslant \frac{2^{2|\nu|+n+2} M^{\prime 2|\nu|+n+1}}{\left(\begin{array}{c}\rho^{\prime} \\ \xi_{0}\end{array}\right)^{|\nu|+n+1}}(|\nu|+n+3) ! k\left|\Omega_{0}\right|(|\nu|=0,1,2, \cdots)$.

Ceci établit que $u(x, t)$ est une fonction analytique en $(x, t)$ dans $(x, t) \in U$, de là il en est de même de la distribution $u(x, t)$ définie par $(3,12)$. Or, $u(x, t)$ est bien la solution de $(1,1)$ avec la donnée initiale :

$$
u(x, 0) \underset{\mathcal{F}}{\longrightarrow} \sum_{i=1}^{N}\left(\sigma_{i}(\omega)\right)_{\xi \geqslant \xi_{0}} R_{i}(\omega),
$$

où $\left(\sigma_{i}(\omega)\right)_{\xi \geq \xi_{0}}$ désigne la fonction qui est zéro pour $\xi<\xi_{0}$ et égale à $\sigma_{i}(\omega)$ pour $\xi \geqslant \xi_{0}$. Pour achever notre démonstration, il ne reste 
qu'à constater l'analyticité de la solution $u_{0}(x, t)$ de $(1,1)$ avec la donnée initiale:

$$
u_{0}(x, 0) \underset{\mathscr{F}}{\longrightarrow} \sum_{i}\left(\sigma_{i}(\omega)\right)_{\xi<\xi_{0}} R_{i}(\omega),
$$

où $\left(\sigma_{i}(\omega)\right)_{\xi<\xi_{0}}$ est la fonction qui est égale à $\sigma_{i}(\omega)$ pour $\xi<\xi_{0}$ et 0 pour $\xi \geqslant \xi_{0}$. Or, ceci est évident, vu que $\mathcal{F}\left(u_{0}(x, t)\right)$ est la solution de l'équation $(1,2)$ et que le support de cette image est toujours comprise dans la boule de rayon $\xi_{0}$ et elle dépend analytiquement en $t$.

4. Nous avons supposé que les racines $\lambda_{i}(\omega)$ sont distinctes. Nous allons montrer que cette hypothèse se généralise, Supposons le système $(1,1)$ hyperbolique au sens que nous avons énoncé au début de l'Introduction. Comme on sait (voir Petrowsky [3], [5], Mizohata [2]), on peut définir, dans ce cas, localement sur la sphère $\Omega_{0}$, l'ensemble de vecteurs propres $R_{11}(\omega), \cdots, R_{1, p_{1}}(\omega) ; R_{21}(\omega), \cdots$, $R_{2, p_{2}}(\omega) ; \cdots ; R_{s 1}(\omega), \cdots, R_{s . p_{s}}(\omega)$ qui sont analytiques en $\omega$, et linéairement indépendants :

$$
\left(\lambda_{i}(\omega) I-A \cdot \omega\right) R_{i j}(\omega)=0 \quad\left(i=1,2, \cdots, s ; j=1,2, \cdots, p_{i}\right) .
$$

On pose la solution cherchée sous la forme

$$
\hat{u}(\omega \xi, t)=\sum_{j=1}^{s} \exp \left(i \lambda_{j}(\omega) \xi t\right)\left\{v_{0}^{(j)}(\omega, t)+\cdots+v_{n}^{(j)}(\omega, t) / \xi^{n}+\cdots\right\} .
$$

Posons

$$
\begin{gathered}
v_{n}^{(i)}(\omega, t)=\sum_{j=1}^{s}\left(\sum_{k=1}^{p_{j}} \sigma_{n}^{(i) j k} R_{j k}\right) \\
B R_{j k}=\sum_{\substack{1 \leqslant h<s \\
1 \leqslant l \leqslant p_{h}}} b_{j k}^{h l}(\omega) R_{h l}(\omega), \text { de là } \\
B v_{n}^{(i)}=\sum_{j k}\left(\sum_{h, l} \sigma_{n}^{(i) h l} b_{j k}^{h l}\right) R_{j k} .
\end{gathered}
$$

Conformément à la formule $(3,5)$, on a

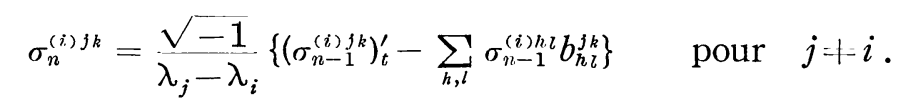

et à $(3,6)^{\prime}$, on a

$$
\begin{aligned}
& \left(\sigma_{n-1}^{(i) i k}\right)_{t}^{\prime}-\sum_{1 \leqslant l \leqslant p_{i}} \sigma_{n-1}^{(i) i l} b_{i l}^{i k}=\sum_{\substack{\{h ; h \rightarrow i\} \\
\left\{l=1, \cdots p_{h}\right\}}} \sigma_{n-1}^{(i) h l} b_{h l}^{i k} \\
& \left(i=1,2, \cdots, s ; k=1,2, \cdots, p_{i}\right), \text { avec la condition initiale }
\end{aligned}
$$




$$
\sigma_{n-1}^{(i) i k}(\omega, 0)=-\sum_{\left\{i^{\prime} ; i^{\prime}, i\right\}} \sigma_{n-1}^{\left(i^{\prime}\right) i k} .
$$

Pour évaluer $\sigma_{n}^{(i) i 1}, \cdots, \sigma_{n}^{(i) i, p_{i}}$ d'après le système d'équation différentielle $(4,6)$, on s'appuie sur le

LEMME 4,1 . Soit $X(t)=\left(x_{1}(t), \cdots, x_{r}(t)\right) t \geqslant 0$, une solution du systeme $X^{\prime}(t)=D X(t)+K(t)$, où $D$ est une matrice d'ordre $r$, on a

où

$$
\begin{aligned}
& |X(t)| \leqslant \exp (|| D|| t)\left(|X(0)|+\max _{0 \leqslant \tau \leqslant t}|K(\tau)|\right), \\
& |X(t)|=\left(\sum_{i}\left|x_{i}(t)\right|^{2}\right)^{\frac{1}{2}}
\end{aligned}
$$

$\|D\|$ est la norme de $D$ considéré comme application de $C^{r}$ dans $C^{r}$.

$$
X^{(p)}(t)=D^{p} X(t)+\sum_{q=0}^{p-1} D^{p-q-1} K^{(q)}(t) .
$$

On aura donc l'évaluation analogue au lemme 3,2 . On aura donc

TheOREME 4,1. Soit $E(x, t)$ une des solutions élémentaires du système hyperbolique $(1,1)$, alors $E(x, t)$ est une fonction analytique (à valeurs vectorielles) en $(x, t)$ sauf sur les bicaractéristiques issues de l'origine.

Enonçons une conséquence du Théorème. Désignons par $S\left(x_{0}, t_{0}\right), t_{0} \neq 0$, l'intersection des lignes bicaractéristiques issues du point $\left(x_{0}, t_{0}\right)$ (du système $(1,1)$ ) avec le plan $t=0$.

THEOREME 4,2. Soit $u(x, t)$ une solution $d u$ systeme $(1,1)$. Si la donnée initiale, $u(x, 0)$, est analytique au voisinage de $S\left(x_{0}, t_{0}\right)$, alors $u(x, t)$ est analytique en $(x, t)$ au voisinage de $\left(x_{0}, t_{0}\right)$.

DÉmonstration. Il suffit de montrer l'analyticité en $x$. En effet, si cela est établi, le théorème de Cauchy-Kowalewsky, combiné avec le théorème de Holmgren (sur l'unicité) nous montre l'analyticité de $u(x, t)$ en $(x, t)$ au voisinage de $\left(x_{0}, t_{0}\right)$.

Soient $E_{1}^{\prime}(x, t), \cdots, E_{N}^{\prime}(x, t)$ le système fondamental de solutions élémentaires du système adjoint à $(1,1)$ :

$$
\left(-\frac{\partial}{\partial t}+\sum_{j}{ }^{t} A_{j} \frac{\partial}{\partial x_{j}}-{ }^{t} B\right) E_{i}^{\prime}(x, t)=0
$$

vérifiant

$$
E_{i}^{\prime}(x, 0)=\left(\begin{array}{c}
0 \\
\vdots \\
\delta_{x} \\
\vdots \\
0
\end{array}\right) i
$$


En désignant par $E_{i}^{\prime}\left(x, t ; x_{0}, t_{0}\right)=\tau_{x_{0}, t_{0}} E_{i}^{\prime}(x, t)$ (translatée de $\left(x_{0}, t_{0}\right)$ de la distribution $\left.E_{i}^{\prime}(x, t)\right)$, on a

$$
\begin{aligned}
u\left(x_{0}, t_{0}\right) & =\sum_{i} \int u_{i}(x, 0) E_{i}^{\prime}\left(x, 0 ; x_{0}, t_{0}\right) d x \\
& =\sum_{i} \int u_{i}(x, 0) E_{i}^{\prime}\left(x-x_{0} ; 0, t_{0}\right) d x
\end{aligned}
$$

où l'intégration est à prendre au sens de distribution.

Ou encore, en désignant $E_{i}(\xi-x)=E_{i}^{\prime}\left(\xi-x ; 0, t_{0}\right), \rho_{i}(x)=u_{i}(x, 0)$,

$$
u(x, t)=\sum_{i} \int \mathcal{P}_{i}(\xi) E_{i}(\xi-x) d \xi .
$$

Remarquons que les bicaractéristiques de $(4,8)$ sont identiques à celles de $(1,1)$.

Pour notre but, il suffit de montrer le

Lemme 4,2. Soit $u(x)$ la distribution définie par

$$
u(x)=\int f(\xi-x) \varphi(\xi) d \xi
$$

On suppose que

i) $f(x)$ est une distribution à support compact, qui est analytique en dehors de l'ensemble fermé $S$;

ii) $\mathcal{P}(x)$ est une distribution, qui est analytique au voisinage de l'ensemble $S_{x_{0}}=\tau_{x_{0}} S$ (translatée de $S$ ).

Alors $u(x)$ est une fonction analytique au voisinage $d u$ point $x_{0}$.

Démonstration. Ce qui va suivre est essentiellement le même que [6] Exposé 6, Proposition 1.

On prend $\alpha(\xi), 0 \leqslant \alpha(\xi) \leqslant 1$, qui vaut 1 au voisinage de $S_{x_{0}}$ dont le support soit contenu complètement dans le domaine de l'analyticité de $\rho(\xi)$. On décompose $u(x)$ en deux :

$$
\begin{aligned}
u(x)=u_{1}(x)+u_{2}(x) & =\int f(\xi-x)[1-\alpha(\xi)] \rho(\xi) d \xi \\
& +\int f(\xi-x) \alpha(\xi) \varphi(\xi) d \xi
\end{aligned}
$$

Sur le support de $[1-\alpha(\xi)] \rho(\xi) \in \mathscr{E}^{\prime}, f(\xi-x)$ est une fonction analytique en $\xi-x$, si $x$ est assez voisin de $x_{0}$. Cela montre que $u_{1}(x)$ est analytique au voisinage de $x_{0}$.

L'analyticité de $u_{2}(x)$ se démontre de la manière suivante: 


$$
D^{\nu} u_{2}(x)=(-1)^{|\nu|} \int D_{\xi}^{\nu} f(\xi-x) \alpha(\xi) \rho(\xi) d \xi=\int f(\xi-x) D^{\nu}[\alpha(\xi) \rho(\xi)] d \xi .
$$

Par Leibniz, elle s'écrit

$$
\int f(\xi-x) \alpha(\xi) D^{\nu} \varphi(\xi) d \xi+\sum_{\mu \neq 0} C_{\mu}^{\nu} \int f(\xi-x) D^{\mu} \alpha(\xi) D^{\nu-\mu} \varphi(\xi) d \xi .
$$

Evaluons le premier terme. $f(x)$ s'exprime

$$
f=\sum_{|p| \leqslant m} D^{p} \mu_{p} \text { où } \mu_{p} \text { sont des mesures à support compact. }
$$

D'autre part, comme $\varphi(\xi)$ est une fonction analytique au voisinage du support de $\alpha(\xi)$, on a

De là

$$
\left|D^{\nu} \varphi(\xi)\right| \leqslant \frac{\nu ! M_{\varphi}}{\rho^{|\nu|}} \text { pour } \xi \in \text { support de } \alpha \text {; on suppose } \rho \leqslant 1 \text {. }
$$

$(4,13)\left|\int f(\xi-x) \alpha(\xi) D^{\nu} \mathcal{P}(\xi) d \xi\right| \leqslant \sum_{|p|<^{m}}\left(\max \left|D^{p}\left[\alpha(\xi) D^{\nu} \mathcal{P}(\xi)\right]\right|\right) \cdot\left|\int\right| d \mu_{p} \mid$ $\leqslant c_{m} \alpha(m) K \frac{(|\nu|+m) !}{\rho^{|\nu|+m}} M_{\varphi}$, où $c_{m}$ est une constante qui ne dépend que de $m ; \alpha(m)=\max _{|p| \leqslant m}\left(\max \left|D^{p} \alpha(\xi)\right|\right) ; \quad K=\max _{|p| \leqslant m} \int\left|d \mu_{p}\right|$.

Considérons les derniers termes:

$$
\int f(\xi-x) D^{\mu} \alpha(\xi) D^{\nu-\mu} \varphi(\xi) d \xi=(-1)^{\mid \mu_{1}} \int \alpha(\xi) D_{\xi}^{\mu}\left[f(\xi-x) D^{\nu-\mu} \varphi(\xi)\right] d \xi,
$$

il faut noter que l'intégration en $\xi$ ne s'étend que sur l'ensemble où $\alpha_{\xi}^{\prime} \neq 0$. Comme $f(\xi-x)$ y est analytique en $\xi-x$, si $x$ est voisin de $x_{0}$, on a

$\left|D_{\xi}^{\nu} f(\xi-x)\right| \leqslant \frac{\nu !}{\rho^{\prime|\nu|}} M_{f} . \quad$ Par Leibniz, $\quad D_{\xi}^{\mu}\left[f(\xi-x) D^{\nu-\mu} \mathcal{T}(\xi)\right]=$ $\sum_{\sigma \leqslant \mu} C_{\sigma}^{\mu} D_{\xi}^{\sigma} f(\xi-x) D^{\nu-\sigma} \mathcal{P}(\xi)$, elle se majore donc par

$\sum_{\sigma \leqslant \mu} C_{\sigma}^{\mu} \frac{\sigma !}{\rho^{\prime|\sigma|}} M_{f} \frac{(\nu-\sigma) !}{\rho^{|\nu-\sigma|}} M_{\varphi} \leqslant \frac{M_{f} M_{\varphi}}{\rho^{\prime \prime \prime^{\nu \mid}}} \sum_{\sigma \leqslant \mu} C_{\sigma}^{\mu} \sigma !(\nu-\sigma) !$, où $\rho^{\prime \prime}=\min \left(\rho, \rho^{\prime}\right)$. Or, $\sum_{\sigma \leqslant \mu} C_{\sigma}^{\mu} \sigma !(\nu-\sigma) !==\mu ! \sum \frac{(\nu-\sigma) !}{(\mu-\sigma) !}$, en majorant tous les termes sous le signe de $\sum$ par $\nu !$, on a $\leqslant \nu ! \sum_{\sigma \leqslant \mu} 1=\nu !\left(\mu_{1}+1\right) \cdots\left(\mu_{n}+1\right) \leqslant(|\nu|+n)$ !. De là,

$(4,14) \quad \mid$ les derniers termes de $(4,12) \mid \leqslant \frac{(|\nu|+n) !}{\rho^{\prime \prime|\nu|}} M_{f} M_{\varphi} 2^{|\nu|}$. 
En ajoutant $(4,13)$ et $(4,14)$, on voit facilement que $D^{\nu} u_{2}(x)$, $\nu \geqslant 0$, sont majorées par une suite vérifiant bien que $u_{2}(x)$ est une fonction analytique.

c.q.f.d.

Comme un cas très particulier du théorème 4,2 , on a le

CoROllaire DU THEORÈm 4,2 . Si la donnée initiale $u(x, 0)$ est analytique en tout $x$, alors la solution $u(x, t)$ est analytique en $(x, t)$ en tous les points $(x, t)$.

5. Il n'est pas difficile de voir que notre résultat est vrai pour les systèmes hyperboliques d'ordre plus haut qu'un. Limitons-nous d'équation hyperbolique :

$$
M[u]=0, \text { où }
$$

$$
\begin{gathered}
M=\left(\frac{\partial}{\partial t}\right)^{m}+\sum_{k_{0}+k_{1}+\cdots+k_{n}=m} a^{(k)}\left(\frac{\partial}{\partial t}\right)^{k_{0}<m}\left(\frac{\partial}{\partial x_{1}}\right)^{k_{1}} \cdots\left(\frac{\partial}{\partial x_{n}}\right)^{k_{n}} \\
+\sum_{k_{0}+\cdots+k_{n} \leqslant m_{-1}} b^{(k)}\left(\frac{\partial}{\partial t}\right)^{k_{0}} \cdots\left(\frac{\partial}{\partial x_{n}}\right)^{k_{n}} .
\end{gathered}
$$

On désigne la partie homogène de degré $m$ de l'image de Fourier de $M$ par $H$ :

$(5,2) \quad H=\left(\frac{\partial}{\partial t}\right)^{m}+\alpha_{1}(\omega)(i \xi)\left(\frac{\partial}{\partial t}\right)^{m-1}+\alpha_{2}(\omega)(i \xi)^{2}\left(\frac{\partial}{\partial t}\right)^{m-2}+\cdots+\alpha_{m}(\omega)(i \xi)^{m}$,

où $\alpha_{i}(\omega)$ sont des fonctions homogènes de degré 0 en $\omega$, qui sont évidemment analytiques en $\omega$.

Posons

$$
\hat{u}_{i}(\omega \xi, t)=(i \xi)^{m-1-i}\left(\frac{\partial}{\partial t}\right)^{i} \hat{u}(\omega \xi, t) \quad(i=0,1,2, \cdots, m-1) .
$$

En désignant par $\hat{v}(\omega \xi, t)=\left(\hat{u}_{0}, \cdots, \hat{u}_{m-1}\right)$, l'équation $\hat{M}[\hat{v}]=0$ s'écrit, en combinant avec $(5,3)$, sous la forme matricielle:

$$
\left[\frac{\partial}{\partial t}-i A(\omega) \xi-B_{0}(\omega)-B_{1}(\omega) / \xi-\cdots-B_{m-1} / \xi^{m-1}\right] \hat{v}(\omega \xi, t)=0
$$

$$
\left.A(\omega)=\mid \begin{array}{rrrrr}
0 & 1 & & & \\
& 0 & 1 & & \\
& & \ddots & & \\
-\alpha_{m}(\omega) & -\alpha_{m-1}(\omega) & \cdots & -\alpha_{1}(\omega)
\end{array}\right) .
$$


Comme on sait, les valeurs propres de $A(\omega)$ sont identiques aux racines du polynome caractéristique de $(5,1)$ :

$$
P(\lambda ; \omega)=\lambda^{m}+\alpha_{1}(\omega) \lambda^{m-1}+\cdots+\alpha_{m}(\omega)=0 .
$$

On suppose que les racines $\lambda_{i}(\omega)$ sont distinctes et réelles pour tout $\omega$. Les $\lambda_{i}(\omega)$ sont alors des fonctions analytiques. Dans $(5,4)$, $A(\omega), B_{0}(\omega), \cdots, B_{m-1}(\omega)$ sont des matrices (d'ordre $m$ ) homogènes de degré 0 en $\omega$, et analytiques.

Nous allons construire la solution $\hat{v}(\omega \xi, t)$ de $(5,4)$ vérifiant la condition initiale :

$$
\hat{v}(\omega \xi, 0)=\left(\begin{array}{c}
0 \\
0 \\
\vdots \\
0 \\
1
\end{array}\right)
$$

en visant à la construction de la solution $u(x, t)$ de $(5,1)$ vérifiant

$$
u(x, 0)=\frac{\partial}{\partial t} u(x, 0)=\cdots=\left(\frac{\partial}{\partial t}\right)^{m-2} u(x, 0)=0, \quad\left(\frac{\partial}{\partial t}\right)^{m-1} u(x, 0)=\delta_{x} .
$$

Remarquons, en chemin faisant, que les autres solutions élémentaires vérifiant:

$$
\left(\begin{array}{c}
\partial \\
\partial t
\end{array}\right)^{i} u_{j}(x, 0)=\left\{\begin{array}{lll}
\delta_{x} & \text { pour } i=j \\
0 & \text { pour } i=j-j
\end{array} \quad(0 \leqslant j \leqslant m-2)\right.
$$

se détermineront de la même manière. Par conséquent, nous n'en donnons pas la démonstration.

On pose, comme auparavant

$$
\hat{v}(\omega \xi, t)=\sum_{j=1}^{m} \exp \left(i \lambda_{j} \xi t\right)\left\{v_{0}^{(j)}+\cdots+v_{n}^{(j)} / \xi^{n}+\cdots\right\} .
$$

En posant les coefficients de $\xi^{-(n-1)}$ zéro, on a

$(5,8) \quad i\left(\lambda_{j}(\omega) I-A(\omega)\right) v_{n}^{(j)}=-\left(v_{n-1}^{(j)}\right)_{t}^{\prime}+B_{0} v_{n-1}^{(j)}+B_{1} v_{n-2}^{(j)}+\cdots+B_{m-1} v_{n-m}^{(j)}$ (on pose $v_{-1}=v_{-2}=\cdots=0$ ).

Décomposons la donnée initiale $(5,6)$ :

$$
\left(\begin{array}{c}
0 \\
\vdots \\
0 \\
1
\end{array}\right)=\sum_{j=1}^{m} \sigma_{j}(\omega) R_{j}(\omega) .
$$


Comme dans le cas précédent, on pose

$$
v_{n l}^{(i)}=\sum_{j} \sigma_{n}^{i j}(\omega, t) R_{j}(\omega) .
$$

On pose finalement,

$$
B_{0} v_{n-1}^{(i)}+\cdots+B_{m-1} v_{n-m}^{(i)}=\sum_{j} P_{j}^{(i)}\left(\omega ; \sigma_{n-1}, \cdots, \sigma_{n-m}\right) R_{j},
$$

où $P_{j}^{(i)}$ sont des formes linéaires en $\sigma_{n-1}^{i j}, \cdots, \sigma_{n-m}^{i j}$ à coefficients dans les fonctions analytiques en $\omega$.

Ceci posé, $(5,8)$ s'écrit sous la forme

$$
\sigma_{n}^{i j}=\frac{\sqrt{-1}}{\lambda_{j}-\lambda_{i}}\left\{\left(\sigma_{n-1}^{i j}\right)_{t}^{\prime}-P_{j}^{(i)}\left(\omega ; \sigma_{n-1}, \cdots, \sigma_{n-m}\right)\right\} \quad j \neq i .
$$

En écrivant

$$
P_{i}^{(i)}=b_{i i}(\omega) \sigma_{n-1}^{i i}+Q_{i}^{(i)}\left(\omega ; \sigma_{n-1}^{i j}, \sigma_{n-2}, \cdots, \sigma_{n-m}\right)
$$

où $Q_{i}^{(i)}$ ne contient pas de terme $\sigma_{n-1}^{i i}$, on a

$$
\left(\sigma_{n-1}^{i i}\right)^{\prime}-b_{i i} \sigma_{n-1}^{i i}=-Q_{i}\left(\omega ; \sigma_{n-1}^{i j}, \sigma_{n-2}, \cdots, \sigma_{n-m}\right),
$$

avec la condition initiale

$$
\sigma_{n-1}^{i i}(\omega, 0)=-\sum_{\{j ; j \neq i\}} \sigma_{n-1}^{j i}(\omega, 0) .
$$

Il sera facile de voir qu'on a une inégalité de la forme du Lemme 3 , 2. D'où on voit que l'image réciproque de $\left(\hat{v}_{m-1}(\omega \xi, t)\right)_{\xi \geq \xi_{0}}$ est une fonction analytique en $(x, t)$ où $(x, t)$ n'appartient pas à $(B)$. Prenons les autres composants, par exemple $\left(\hat{v}_{0}(\omega \xi, t)\right)_{\xi \geq \xi_{0}}$. Il en est de même de l'image réciproque de $(i \xi)^{-(m-1)}\left(\hat{v}_{0}(\omega \xi, t)\right)_{\xi \geqq \xi_{0}}$. Ainsi, on voit que l'image réciproque de $\left(\hat{u}(\omega \xi, t), \cdots,\left(\begin{array}{c}\partial \\ \partial t\end{array}\right)^{m-1} \hat{u}(\omega \xi\right.$, $t))_{\xi \geq \xi_{0}}$ est analytique en $(x, t)$ dans l'ouvert complémentaire de $(B)$. En ajoutant l'image réciproque de $\left(\hat{u}(\omega \xi, t), \cdots,\left(\frac{\partial}{\partial t}\right)^{m-1} \hat{u}(\omega \xi, t)\right)_{\xi<\xi_{0}}$, on voit que $u(x, t)$ est une fonction analytique en $(x, t)$ dans l'ouvert envisagé.

\section{BIBLIOGRAPHIE}

[1] P. D. Lax, Asymptotic solutions of oscillatory initial value problems, Duke Math. J. 24 (1957), p. 627-646.

[2] S. Mizohata, Problème de Cauchy pour les systèmes hyperboliques et paraboliques, à paraître. 
[3] I. G. Petrowsky, Über das Cauchysche Problem für ein System linearer partieller Differentialgleichungen im Gebiete der nicht-analytischen Funktionen, Bull. de l'Univ. de l'Etat de Moskou (1938), p. 1-74.

[4] - On the diffusion of waves and the lacunas for hyperbolic equations, Rec. Math. (Math. Sbornik), 59 (1945), p. 289-370.

[5] - Quelques remarques sur mes travaux relatifs au problème de Cauchy (en russe), Ibid. 39 (1956), p. 267-272.

[6] L. Schwartz, Séminaire sur les équations aux dérivées partielles (1954-55), Paris. 